\title{
Physical Playlist: Bringing Back the Mix-Tape
}

\author{
Daniel Burnett ${ }^{1}$, Adrian Gradinar ${ }^{1}$, Joel Porter $^{1}$, Mike Stead $^{1}$, Paul \\ Coulton $^{1}$, Ian Forrester ${ }^{2}$ \\ ${ }^{1}$ ImaginationLancaster, LICA Building, Lancaster University, Lancaster, UK, LA1 4YW \\ \{d.burnett, a.gradinar, j.porter, m. stead, p. coulton $\}$ lancaster.ac.uk \\ ${ }^{2}$ Dock House, MediaCity, Salford, UK \\ ian.forrester 1 @bbc.co.uk
}

\begin{abstract}
To those of a certain age the concept of the mix-tape holds fond memories, and generally not of the musical content they contained, but rather the emotional and physical connection they represented with either its creator or recipient. They provided an embodiment of the time and effort it its creation and thus presented the same qualities of other handmade gifts. The advent of digital content, and particularly the $\mathrm{mp} 3$, for storage and streaming meant that audio content could be shared more quickly and easily than ever before. However, the creation of a digital playlist does not embody the same qualities present in a mix-tape and thus has not gained the same cultural significance. This research re-imagines the mix-tape for digital content as physical customizable jewellery that can once again embody values not generally attributed to digital content. Through a discussion of the design process and the results of preliminary evaluation, the potential benefits on the user experience of sharing digital content through physical objects have been highlighted.
\end{abstract}

Keywords. Tangible, Embodied, NFC, Customizable, Jewellery

\section{Introduction}

The shared mix-tape had an emotional and physical connection for people that digitally shared content often lacks. This connection comes from the fact that objects or artefacts often symbolize something more than their intrinsic value, and this is often preserved over the years. Our personal associations with objects often gain subjective meaning based on the memories that we have about them, although such memories are generally hidden and intangible [2]. Where once time was spent deco- 
rating the cover of the mix-tape ${ }^{1}$ and carefully cultivating the tracks and possibly taking upwards of an hour to make, it now takes mere seconds with digital services such as Spotify, where on average nine playlists are created each second ${ }^{2}$. Between the mix-tape and Spotify was the writeable $\mathrm{CD}$ but they came too late, or too close, to the emergence of the mp3 to become a shareable treasured object akin to the mix-tape. The project presented in this research and developed in conjunction with BBC R\&D at Media City UK resulted in the creation of a system, The Physical Playlist, that can be used to explore users experience of a physical shareable personalized object that has digital content embedded within it. Thus the project differs from those that have used objects as a means of storing memories, such as Memory Boxes [4] or Stevens et al's Living Memory Box [9], as the object itself primarily represents the embodiment of the time and effort the creator took to personalize the gift thus making it more meaningful than a purely digital playlist.

\section{Design Process}

This research is part of a larger $\mathrm{AHRC}^{3}$ project The Creative Exchange ${ }^{4}$ whose aim is to bring together academics and businesses to improve the way they work together through a series of workshops exploring different themes around the concept of The Digital Public Space [7]. The theme of the workshop from which this project emerged was "Making the Digital Physical" and the Physical Playlist was one of the ideas generated by the group who went on to develop the project. The core idea was to recreate the concept of the mix-tape using digital content associated with physical objects. The physical objects could take almost any form imaginable although the original idea was for customizable jewellery and its format chosen for the initial prototype presented in this paper.

As the design was to be created collaboratively it was felt a 'research through design' [3] approach would be adopted, as it would allow all parties to reflect upon the design in an agile and emergent manner. One

\footnotetext{
${ }^{1}$ A mix-tape is the name given to any compilation of songs recorded onto any audio format although it is primarily associated with the compact audiocassette. https://press.spotify.com/uk/information/ Arts and Humanities Research Council http://www.thecreativeexchange.org/
} 
of the earliest considerations was how to embed digital content within an object that could be used easily by a user. The obvious solution to this was Near Field Communications (NFC) tags as they are relatively cheap, come in a variety of form factors, and now can be easily programmed via a mobile phone [1].

After deciding upon NFC tags as the bearer of the playlist, a method of personalizing the tags and then reading them back to play the associated media would have to be created. The tags chosen needed to be relatively small and robust, in the end a $14 \mathrm{~mm}$ laundry tag was settled upon. Given its resilient nature and small footprint it was ideal for the application as the jewellery it is attached to may be worn out and about in diverse environments.

While different ideas for embedding the tag emerged a design was settled on that would allow easy programming of the tag and individual customization when incorporated within a piece of jewellery, which in this case was a bracelet. This resulted in the definition of a widget to hold the required tag and after a number of design iterations, shown in Fig.1, a widget was produced that fulfilled this purpose.

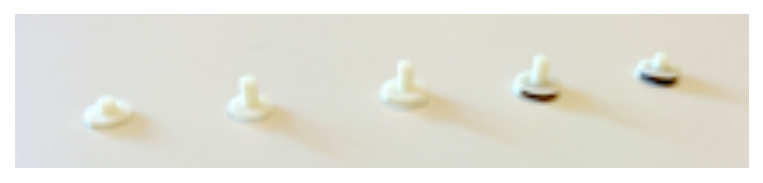

Fig. 1. Prototypes of widget from left (initial) to right (final).

With the NFC tags suitably housed the next step was to create a means to turn this individual item into a playlist. A simple bracelet was chosen as shown in Fig. 2 below, as each widget could then be customized to produce something akin to a charm bracelet.

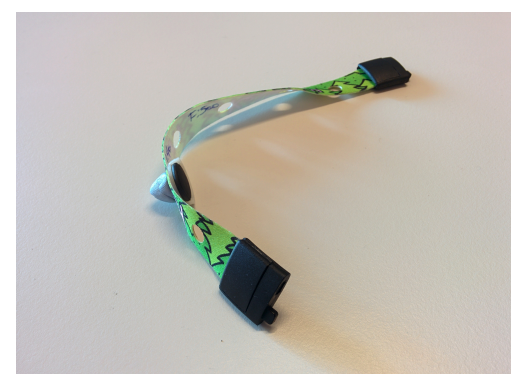

Fig. 2. Original prototype bracelet with an NFC tag embedded in a widget 
With the playlist form factor created a method for embedding content and then reading and playing the tracks was required.

\subsection{Embedding Content within Tags}

An elegant solution was required for the process of storing the track data within the tag, as the chosen tags were only capable of storing 48 bytes. A simplified protocol was created where the first part of the data would be the type of track i.e. $s$ for Spotify, $y$ for YouTube and $i$ for iPlayer, followed by a comma and finished with the URI (Unique Resource Identifier) for that track. The whole string was then enclosed within chevrons to give an indication of the start and end of the tag. For example:

<s, 6JEK0CvvjDjjMUBFOXShNZ>

The most viable option was to create a mobile app for the Android platform due to its open nature and the prevalence of NFC technology already contained within a large number of the phones running the operating system.

With the player capable of playing a multitude of different media types from different services, in this project the media to be embedded came from YouTube ${ }^{5}$, Spotify $^{6}$, and BBC iPlayer $^{7}$ and the developed app had to accommodate all of these in one simple interface. The most effective way to do this was to provide a single text entry space with the option to then search any of the three services; the interface is shown in Fig. 3.

\footnotetext{
${ }^{5} \mathrm{http}: / / \mathrm{www}$. youtube.com/

${ }^{6} \mathrm{https}: / /$ www.spotify.com/

${ }^{7} \mathrm{http}: / /$ www.bbc.co.uk/iplayer/
} 


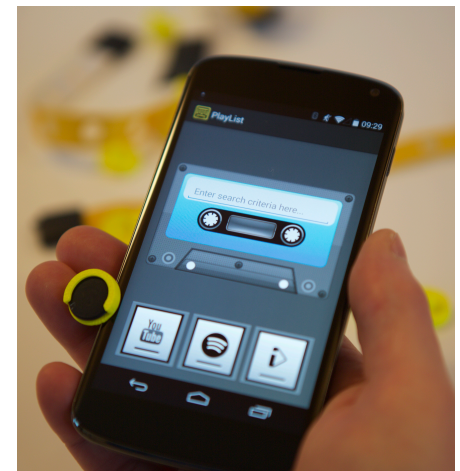

Fig. 3. The Android Application, Buttons can be seen for the three services.

Of course the utility of the App is not limited only to the initial writing of the data to the tag, it can also be used to re-write the tag so playlists can be edited on the fly to personalize them even further.

Once a playlist has been created the next step would be to play it back, for this a working device that can read the NFC tags and create a playlist and ultimately play the tracks was required.

\subsection{Playing the Tracks}

For the design team maintaining the order of the playlist was crucial and so it was decided that any player developed would have to respect the creators' order of the playlist. Working from a position of slow technology $[5,6]$ the process of building the playlist and the act of placing the tags into the bracelet then fixing them in place, creates a time for self reflection and allows the creator to contemplate how best to actually curate the playlist.

From this standpoint a player design was formed that read the bracelet from top to bottom through the use of a rotating rod that moves a platform which can be seen in the left most image in Fig. 4 below. This image also shows the subsequent refined design showcased at MozFest $2014^{8}$ in the middle and the 3D model that has become the finalised design.

\footnotetext{
${ }^{8} \mathrm{http} / / / 2014$.mozillafestival.org
} 

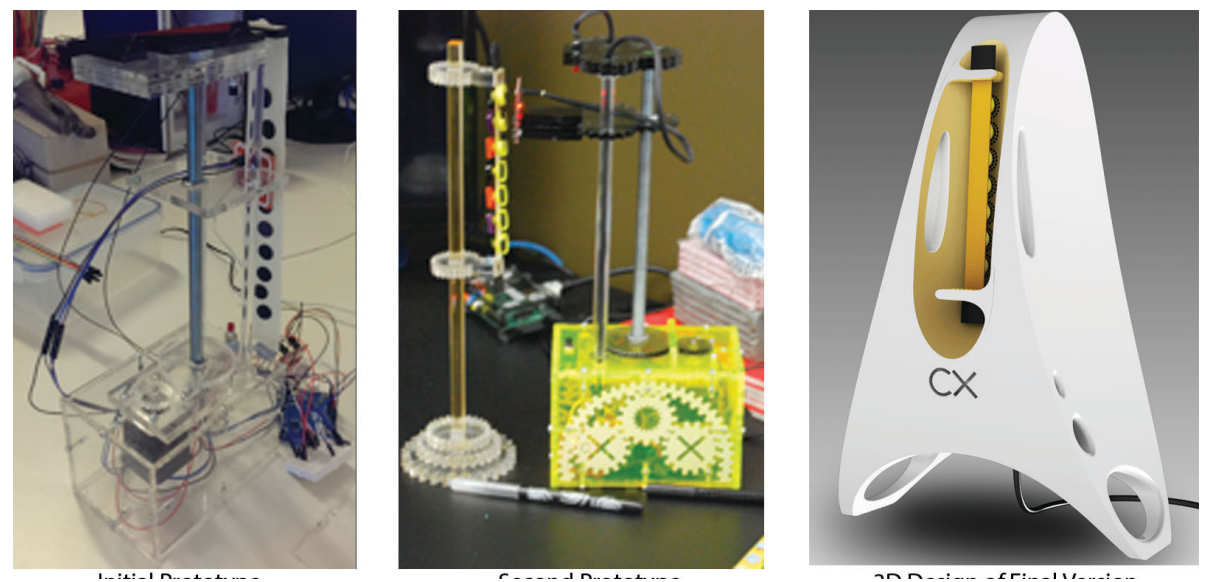

Fig. 4. Initial prototype to Proposed Final Player (Left to Right)

As for the actual playback of the playlist itself, the player, is in fact an Arduino ${ }^{9}$ connected to a RaspberryPi ${ }^{10}$ and transmits the data read from the tag via serial connection where it is then decoded and added to the playlist stored on the RaspberryPi. The playlist begins to play when the first tag is scanned and the arm continues to move down the playlist scanning until it encounters the next unread tag, this is then added to the playlist and the player ceases its movement until the previous track is completed. This is slow technology as was originally coined but also represents its more recent consideration as a way of slowing down the consumption of media [8]. The playback screen on the monitor is shown in Fig. 5.

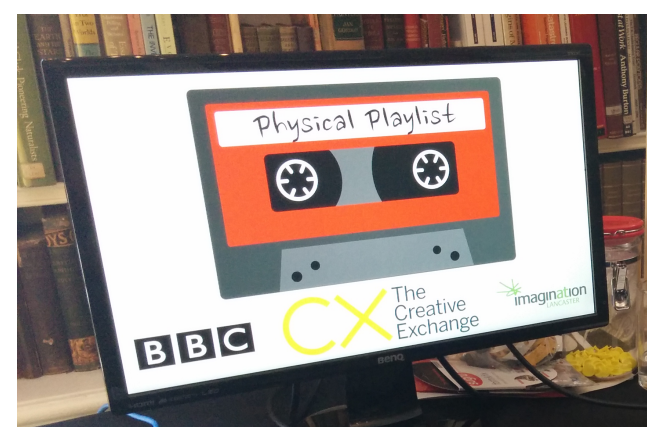

Fig. 5. Playback screen of the Physical Playlist.

\footnotetext{
9 http://www.arduino.cc/

$10 \mathrm{http}: / / \mathrm{www}$.raspberrypi.org
} 


\section{Initial Prototype Evaluation}

The customizable physical shareable and a bespoke media content player were taken to MozFest 2014 for preliminary evaluation where participants were invited to create and play their own bracelets. At the end of the exercise 24 of the participants completed a simple visual tool to indicate how they currently share data and how likely they are to share that data in the future with different peer groups. The tool was designed to encourage comment and debate and enabled the researchers not only to ascertain that the users were able to create and play the produced objects easily it also drew information from the users which suggested that in the future they would be more likely to share a larger amount of personal information with friends and family if it were embedded in a physical object compared to current social network platforms.

In terms of the acceptance of slow technology perhaps surprisingly the younger participants enjoyed that they couldn't skip a track and had to listen in order whereas the older participants wanted to rush through and get to the next part faster. These initial insights allowed us to move forward in the design process towards the forthcoming iterations discussed in the following section.

\section{$4 \quad$ Future Work}

The forthcoming stage of the project the 3D modelled version of the reader, shown in Fig. 4, will be created along with some custom made and designed bracelets to give the whole project a cleaner aesthetic and allow users to see the potential of the objects created. Further, plans are also underway to run a series of more in depth workshops with a range of potential user demographics through which a detailed analysis can be produced of how physical embodiment of digital content may affect users relationship with that content.

\section{Conclusions}

Whilst the original mix tapes offered elements of personalization, the flexibility of the potential objects that can be combined to form a physical playlist is considerably greater as they can take almost any form. Further, as the playlist is digitally enabled they can also be made to use 
other information, for instance they could be made so that they could only be played on a specific day, such as a birthday, or at a specific time, or when the weather is warm and sunny, thus allowing the creator to produce a very unique personalized experience.

Overall it is believed that the advent of 3D printing coupled with readily available means of embedding digital content in physical objects has enormous potential in creating new ways of sharing and this project offers a glimpse of the potential of this.

\section{Acknowledgments}

The research presented in this paper has been made possible through the support of a number of organizations most notably the Arts and Humanities Research Council (AHRC) project The Creative Exchange at Lancaster University and BBC Research and Development at Media City UK.

\section{References}

1. Coulton, P., Omer, R., \& William, B. (2006). Experiencing 'touch' in mobile mixed reality games. GDTW 2006 The Fourth International Game Design and Technology Workshop and Conference. (pp. 68-75). Liverpool: Liverpool John Moores University.

2. Csikszentmihalyi, M., \& Halton, E. (1981). The Meaning of Things Domestic Symbols and the Self. Cambridge: Cambridge University Press.

3. Frayling, C. (1993). Research in Art and Design. Royal College of Art Research Papers , 1 (1), 1-9.

4. Frohlich, D., \& Murphy, R. (2000). The Memory Box. Personal and Ubiquitous Computing , 4 (4), 238-240.

5. Hallnas, L., \& Redstrom, J. (2001). Slow Technology - Designing for Reflection. Personal and Ubiquitous Computing, 5 (3), 201-212.

6. Hallnas, L., Jaksetic, P., Ljungstrand, P., Redstrom, J., \& Skog, T. Expressions: Towards a Design Practice of Slow Technology. Proceedings of IFIP INTERACT01: HumanComputer Interaction 2001, (pp. 447-454). Tokyo.

7. Hemment, D., Thompson, B., de Vicente, J., \& Cooper, R. (Eds.). (2013). Digital public spaces. FutureEverything.

8. Odom, W., Banks, R., Durant, A., Kirk, D., \& Pierce, J. Slow Technology: Critical Reflection and Future Directions. DIS '12 Proceedings of the Designing Interactive Systems Conference (pp. 816-817). New York: ACM.

9. Stevens, M. M., Abowd, G. D., Truong, K. N., \& Vollmer, F. (2003). Getting into the Living Memory Box: Family archives \& holistic design. Personal and Ubiquitous Computing , 210-126. 